\title{
Evolution of rattling particles in deviatoric shear deformation of granular material
}

\author{
Lisha Luo $^{1,2}$, Zhifu Shen ${ }^{2 *}$, Xudong Wang ${ }^{2}$, and Hongmei Gao ${ }^{2}$ \\ ${ }^{1}$ School of Construction Engineering, Jiangsu Open University, Nanjing 210036, China \\ ${ }^{2}$ School of Transportation Engineering, Nanjing Tech University, Nanjing 210009, China
}

\begin{abstract}
Granular material such as clean sand in geotechnical engineering is characterized by structured internal deformation pattern and some interesting particle arrangement patterns. This study focuses on the evolution of the fraction of rattling particles in deviator deformation until the critical state. Numerical simulations using the discrete element method reveal the presence of rattling particles (with zero or only one contact with neighbouring particles) even in a very dense packing system. The results show that the initial fraction of rattling particles depends on sample density and particle size distribution. With the increase of deviator strain, the number and volume fractions of rattling particles gradually approach a steady critical state from either a loose or a dense starting point. An effective void ratio, which is calculated by treating rattling particles as voids, can be viewed as new state parameter describing the effective packing density of sands. Besides, the rattling behaviour strongly depends on particle size distribution.
\end{abstract}

\section{Introduction}

The existence of rattling particles has been recognized through discrete element method (DEM) simulations [1]. The concept of particle rattling has been explored theoretically and employed to explain experimental results of granular sands with gapped particle size distribution [2-5]. The major idea in these theories for fine-coarse mixture of granular material is to assume that smaller particles float (as rattling particles) in the voids formed by the skeleton of larger particles. The mechanical contribution of smaller particles to the static and dynamic behaviour of fine-coarse mixture depends on the content and size of smaller particles relative to the larger ones. Following this idea, the inter-grain state concept has been widely adopted to interpret the observed behavior of sands, e.g. in [6,7]. Starting from an idealized binary packing system of granular particles, these theories often assume that: (1) only two distinct particle sizes exist in the system; (2) the particle size disparity is large enough; and (3) the packing condition of the coarse particles is unaffected by the presence of the fine particles, and vice versa. Thus, by neglecting the fines, an index known as the intergranular void ratio, or also widely known as the skeleton void ratio, was used as an alternative to characterize the state of the mixtures of fines and coarse grains [7-9]. A more general case is to have a fraction of the fines participating in force transferring. Thus, the effect of fines is considered by introducing an alternative equivalent skeleton void ratio to replace the skeleton void ratio. However, to fit against experimental data, some semi-empirical functions have to be introduced to characterize the effects of fine particles $[9,10]$.
In fact, even in relatively uniform (compared with the binary fine-coarse mixture) granular material, the presence of rattling particles is ubiquitous. Although rattling particle is a well-known phenomenon in granular material community, there is still a gap between the theoretical understanding and the application in geomechanics community. We may need further work to promote the idea of effective void ratio (or those previous terms such as intergranular void ratio, equivalent skeleton void ratio) for its general application in geomechanics community to describe the density state of granular soil of various types of particle size distribution, from gapped one to uniform one.

This study will examine the phenomenon of particle rattling for granular material with different particle size span with DEM simulations.

\section{DEM simulations of triaxial loading of clean sand}

Samples with three different particle size distributions (referred to as PSD1, PSD2 and PSD3) have been simulated, as shown in Fig. 1. The three PSDs, representing continuous spans of particle sizes with $d_{\max } / d_{\min }$ from 1.35 to 7 . The coefficient of curvature, defined as $\left(d_{30}\right)^{2} /\left(d_{10} d_{60}\right)$, ranges from 1.05 to 1.9 , and the coefficient of uniformity, defined as $d_{60} / d_{10}$, ranges from 1.16 to 3.31 . Here, $d_{10}$ is a soil mechanics terminology meaning that particles with a diameter smaller than $d_{10}$ constitute 10 percent of a soil sample by mass or solid volume. The same applies to $d_{20}, d_{30}$ and $d_{60}$ through this paper).

* Corresponding author: zhifu.shen@ njtech.edu.cn

A video is available at https://doi.org/10.48448/bahw-6d19 


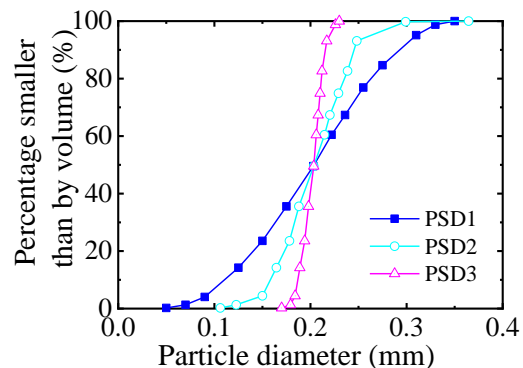

Fig. 1. Particle size distributions used in the simulations.

The rolling and twisting resistance model for clean sand were used to model the contact behavior [11]. In this contact model, two spheres were assumed to physically interact over a circular contact area and rolling moment and torque can be transmitted in addition to the normal and tangential interactions.

The contact behavior formulations are listed in Table 1.The following parameters were chosen according to a parametric study in [11], which can replicate quantitatively the macroscopic behavior of clean sand: $E_{\mathrm{p}}=0.7 \mathrm{GPa}$ (equivalent contact modulus), $\xi=5$ (ratio of normal stiffness to tangential stiffness), $\mu=0.5$ (interparticle friction coefficient), $\beta_{\mathrm{c}}=0.15$ (particle shape parameter used to consider the mechanical effects of particle angularity), $\zeta_{\mathrm{c}}=4$ (local crushing parameter, crushing not considered when equal to 4). Using this contact model, the shape effects of real sand particle (with low aspect ratio) on bulk behavior can be captured with spherical particles. Please refer to [11] for more details of this contact model. The contact model was implemented in PFC3D software [12] for numerical simulations.

Table 1 Summary on the formulations of contact behavior.

\begin{tabular}{|c|c|c|c|}
\hline Interaction & Contact response & Stiffness & Resistance \\
\hline Normal & $\boldsymbol{F}_{\mathrm{n}}=K_{\mathrm{n}} u_{\mathrm{n}} \boldsymbol{n}$ & $K_{\mathrm{n}}=2 E_{\mathrm{p}} R$ & - \\
\hline Tangential & $\boldsymbol{F}_{\mathrm{s}} \leftarrow \boldsymbol{F}_{\mathrm{s}}+K_{\mathrm{s}} \dot{\boldsymbol{u}}_{\mathrm{s}} \Delta t$ & $K_{\mathrm{s}}=K_{\mathrm{n}} / \xi$ & $\mu F_{\mathrm{n}}$ \\
\hline Rolling & $\boldsymbol{M}_{\mathrm{r}} \leftarrow \boldsymbol{M}_{\mathrm{r}}+K_{\mathrm{r}} \dot{\boldsymbol{\omega}}_{\mathrm{r}} \Delta t$ & $K_{\mathrm{r}}=0.25 K_{\mathrm{n}} \beta_{\mathrm{c}}^{2} R^{2}$ & $\zeta_{c} F_{\mathrm{n}} \beta_{\mathrm{c}} R / 4$ \\
\hline Twisting & $\boldsymbol{M}_{\mathrm{t}} \leftarrow \boldsymbol{M}_{\mathrm{t}}+K_{\mathrm{t}} \dot{\boldsymbol{\omega}}_{\mathrm{t}} \Delta t$ & $K_{\mathrm{t}}=0.5 K_{\mathrm{s}} \beta_{\mathrm{c}}^{2} R^{2}$ & $0.65 \mu F_{\mathrm{n}} \beta_{\mathrm{c}} R$ \\
\hline
\end{tabular}

A total of 20,000 particles were used in each sample. A sample with 40,000 particles were also simulated, showing that 20,000 particles are enough for the purpose in this study. The cubic DEM samples with different initial void ratios were prepared by radius expansion method to arrive at an isotropic microstructure. Then, the rigid boundary walls were moved to achieve a desired isotropic stress state. Finally, triaxial compression tests under a confining pressure of $50 \mathrm{kPa}$ were simulated.

Two groups of simulations were run in this study. Group 1 simulation studies the effects of sample density using PSD2 samples with initial void ratios of $e_{0}=0.67$ (dense sample) and 0.94 (loose sample), or initial solid fractions of 0.599 and 0.515 , respectively. Group 2 simulation studies the effects of particle size distribution using PSD1, PSD2 and PSD3 samples, with an initial void ratio of 0.67 (or initial solid fraction of 0.599 ).

\section{Simulation results}

\subsection{Effects of sample density}

Fig. 2 (a) presents typical hardening and softening behaviour of loose and dense sands, respectively, which are accompanied with contraction and dilation, respectively, as shown in Fig. 2 (b). The geometrical arrangement of particles in a sample allows some particles to have zero or only one contact with neighbouring particles, which are called rattling particles. An effective void ratio is defined by treating rattling particles as voids. In Fig. 2 (b), the effective void ratio of the loose sample is much higher than that of the dense sample. With large enough deviator strain, their effective void ratios reach the same steady critical state. Fig. 2 (c) shows that the number fraction of these rattling particles is initially lower in a dense sample $(0.215)$ than in a loose sample (0.376). With an increase in deviator strain, rattling particle fraction in the loose sample decreases while it increases in the dense sample, and finally both samples reach a unique steady state. The same trends are observed for the volume fractions of rattling particles in dense and loose samples as shown in Fig. 2 (d)

Note that the volume fraction of rattling particles is much lower than the number fraction of rattling particles in Fig. 2, which implies that particles of different sizes may have distinct chance to be rattling particles. To investigate this phenomenon, Fig. 3 gives the size distributions of rattling particles at various loading states (with different deviator strains). The size distributions of rattling particles are almost independent of the shear deformation magnitude. Compared with the size distribution of all particles, there exists a threshold particle size $d_{0}$; particles with a diameter smaller than $d_{0}$ have a higher chance to behave as rattling particles than particles with a diameter larger than $d_{0}$. For PSD2 samples simulated here, $d_{0}$ is approximately equal to $d_{30}$ and this $d_{0}$ is independent of sample density.

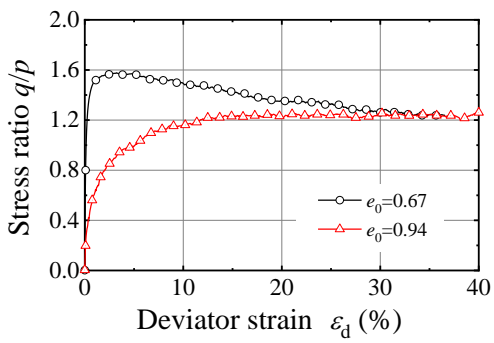

(a)

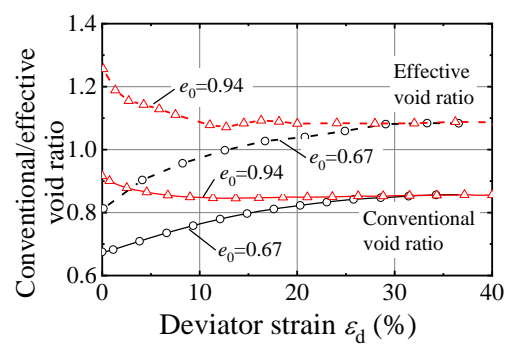

(b) 


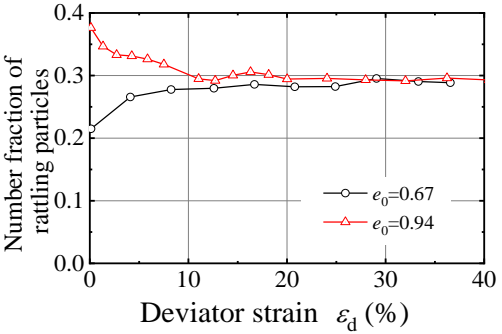

(c)

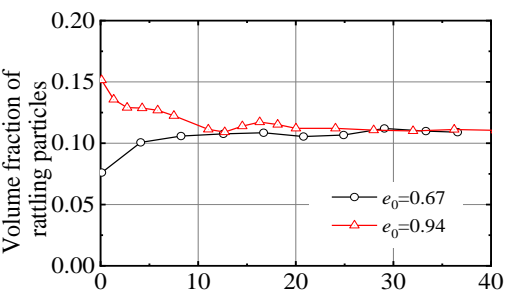

(d)

Deviator strain $\varepsilon_{\mathrm{d}}(\%)$

Fig. 2. Effects of sample density on mechanical responses of clean sand (PSD2): (a) stress ratio, (b) conventional and effective void ratios, (c) number fraction of rattling particles, and (d) volume fraction of rattling particles.

(a)

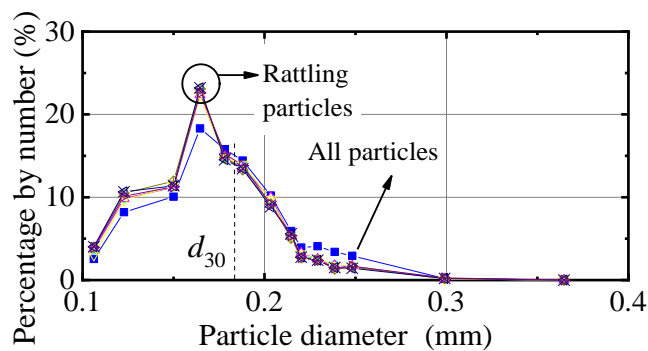

(b)

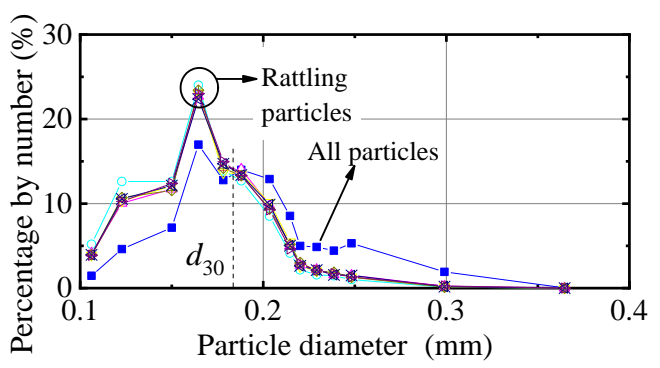

Fig. 3. Size distributions of rattling particles for PSD2 samples: (a) $e_{0}=0.94$ and (b) $e_{0}=0.67$.

\subsection{Effects of particle size distribution}

Fig. 4 presents the mechanical responses and rattling particle fractions of clean sand samples with different PSDs. The general behavior is the same for the three examined PSDs. The widening of particle size brings down the peak stress ratio and enables the sample to be less dilative, as shown in Fig. 4 (a) - (b). Although the three samples with different PSDs have the same initial conventional void ratio, their effective void ratios are quite different, leading to distinct stress-strain curves. The PSD1 sample shows the highest initial effective void ratio among the three while the PSD2 and PSD3 samples have very close initial effective void ratios, which explains the less pronounced softening of PSD1 sample and the close responses of PSD2 and PSD3 samples.

Fig. 4 (c) - (d) show that: (1) both the number and volume fractions of rattling particles are the lowest for PSD3 that has the least particle size span; (2) both fractions increase nonlinearly with deviator strain and finally reach a stable critical state. It is interesting to note that both fractions vary only slightly for PSD1 sample, seemingly indicating that the rattling behavior has been predefined by the initial particle arrangement; this needs further study.

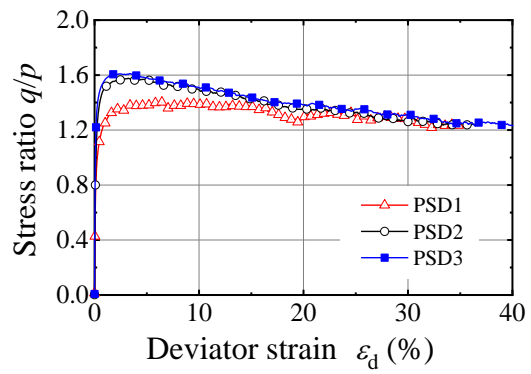

(a)

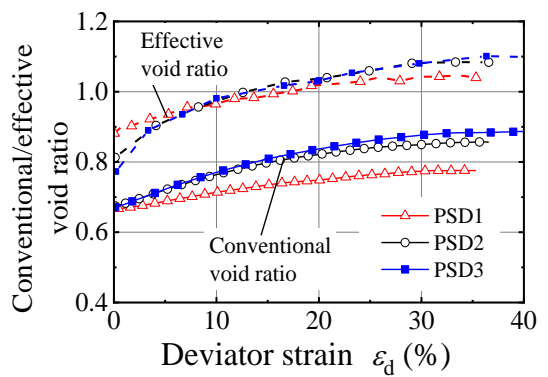

(b)

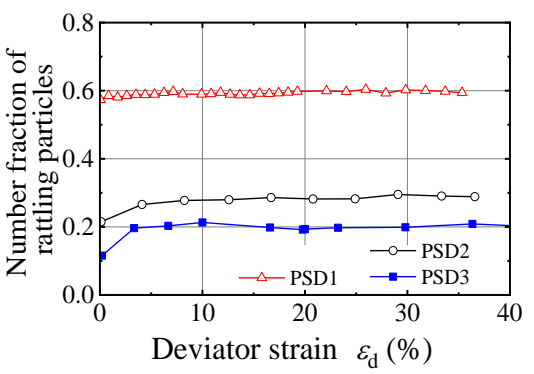

(c)

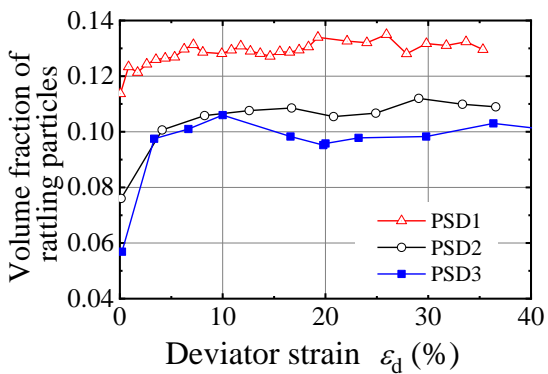

Fig. 4. Effects of particle size distribution on mechanical responses of clean sand ( $\left.e_{0}=0.67\right)$ : (a) stress ratio, (b) conventional and effective void ratios, (c) number fraction of rattling particles, and (d) volume fraction of rattling particles.

Fig. 5 shows that the threshold particle size $d_{0}$ is approximately equal to $d_{20}$ for PSD1, $d_{30}$ for PSD2 and $d_{60}$ for PSD3. The difference between rattling particle size distribution and particle size distribution of all particles becomes less significant when the particle size 
range reduces from in PSD1 to in PSD3. That is, when the particle size in an assembly tends to be uniform, particles of each size tend to have the same chance to be candidates of rattling particles; otherwise, smaller particles are preferred as rattling particles.

(a)

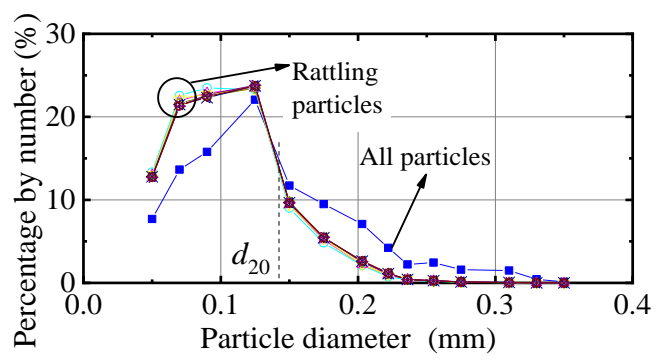

(b)

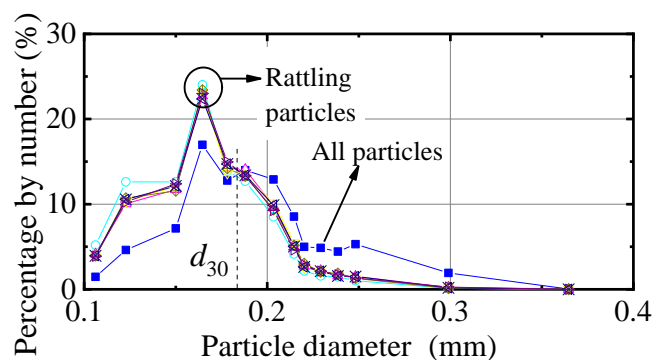

(c)

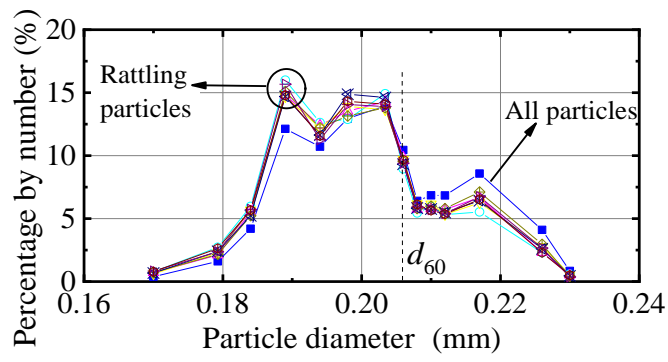

Fig. 5. Size distributions of rattling particles for different PSDs $\left(e_{0}=0.67\right)$ : (a) PSD1, (b) PSD2, and (c) PSD3.

\section{Concluding remarks}

The purpose of this paper is to present some numerical simulation results, focusing on rattling particles in deviatoric loading, and to initiate discussions on the implication of particle rattling in geomechanics.

Particle rattling is a general phenomenon in stressed granular material, such as clean sand in geotechnical engineering. That is, some particles float in the voids formed by other particles when an assembly is subjected to external loads. The effective void ratio, which treats rattling particles as voids since they take no loading, can be viewed as a new density state parameter. Both the number and volume fractions of rattling particles increase (decrease, respectively) with deviator strain for dense (loose, respectively) samples and finally reach the same steady critical state. The rattling behavior strongly depends on particle size distribution of clean sands, which can be captured by a threshold particle size $d_{0}$ that delimits the size range of distinct participation tendency of rattling. With additional simulations (not shown here), it is found that $d_{0}$ does not change with stress level, stress path and sample density, but it strongly depends on particle size distribution. Therefore, $d_{0}$ may be regarded as a characteristic particle size for a specific particle size distribution.

Further study is needed to examine the effects of gapped particle size (i.e., discontinuous particle size distribution), and the effects of particle shape. Then, construction of a predictive model of rattling fraction and effective void ratio based on particle size distribution is possible, which finally can be inserted into a micromechanics based constitutive model for sand in geomechanics. The macroscopic phenomenological constitutive model of sand may also incorporate the effective void ratio, rather than conventional void ratio, in hope of simplifying the constitutive functions, such as stress-dilatancy relation. This would be promising in theoretical geomechanics since the effective void ratio is an easy-to-use scalar quantity and it does shed light upon an important aspect of the microscopic arrangement of particles under deviatoric loading.

The research is funded by Natural Science Foundation of the Jiangsu Higher Education Institutions of China with Grant No. 19KJB560015, National Natural Science Foundation of China for Young Researchers with Grant No. 51908284, and Natural Science Foundation of Jiangsu Province for Young Researchers with Grant No. BK20190667, which are sincerely appreciated. The authors would like to thank Prof. Mingjing Jiang in Tianjin University, China for his support in numerical simulations.

\section{References}

[1] C. Thornton, Geotechnique 50, 43-53 (2000)

[2] M. M. Rahman, S. R. Lo, Geomech. Geoeng. 3, 179-190 (2008)

[3] S. Thevanaygam, S. Mohan, Geotechnique 50, 1$23(2000)$

[4] L. Zuo, B. A. Baudet, Soils Found. 5, 213-219 (2015)

[5] S. L. Yang, S. Lacasse, R. Sandven, Geotech. Test. J. 29, 102-110 (2006)

[6] W. J. Chang, C. W. Chang, J. K. Zeng, Soil Dyn. Earthquake Eng. 56, 74-85 (2014)

[7] G. X. Chen, Q. Wu, T. Sun, K. Zhao, E. Q. Zhou, L. Y. Xu, Y. G. Zhou. J. Earthquake Eng. 25, 1-34 (2021)

[8] S. Thevanayagam, G. R. Martin, Soil Dyn. Earthquake Eng. 22, 1035-1042 (2002)

[9] M. M. Rahman, S. R. Lo, M. A. L. Baki, Acta Geotech. 6, 183-194 (2011)

[10] A. Mohammadi, A. Qadimi, Acta Geotech. 10, 587-606 (2015)

[11] M. J. Jiang, Z. F. Shen, J. F. Wang, Comput. Geotech. 65, 147-163 (2015)

[12] Itasca Consulting Group Inc. PFC3D (Particle Flow Code in Three Dimensions) user's guide (version 6.0). Minneapolis, America (2019) 